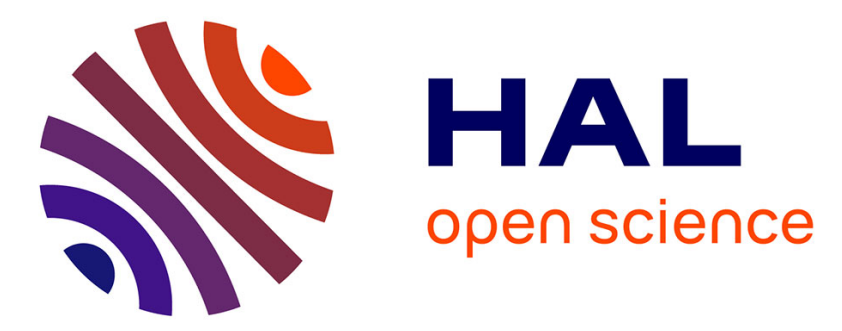

\title{
Optimal Life-History Strategy Differs between Philopatric and Dispersing Individuals in a Metapopulation
}

\author{
Olivier Cotto, Alexander Kubisch, Ophélie Ronce
}

\section{- To cite this version:}

Olivier Cotto, Alexander Kubisch, Ophélie Ronce. Optimal Life-History Strategy Differs between Philopatric and Dispersing Individuals in a Metapopulation. American Naturalist, 2014, 183 (3), pp.384-393. 10.1086/675064 . hal-03119929

\section{HAL Id: hal-03119929 \\ https://hal.science/hal-03119929}

Submitted on 25 Jan 2021

HAL is a multi-disciplinary open access archive for the deposit and dissemination of scientific research documents, whether they are published or not. The documents may come from teaching and research institutions in France or abroad, or from public or private research centers.
L'archive ouverte pluridisciplinaire HAL, est destinée au dépôt et à la diffusion de documents scientifiques de niveau recherche, publiés ou non, émanant des établissements d'enseignement et de recherche français ou étrangers, des laboratoires publics ou privés.

\section{(c) (1) $\$$}

Distributed under a Creative Commons Attribution - NonCommerciall 4.0 International 


\title{
Optimal Life-History Strategy Differs between Philopatric and Dispersing Individuals in a Metapopulation
}

\author{
Olivier Cotto, ${ }^{\star}$ Alexander Kubisch, and Ophélie Ronce \\ Université Montpellier 2, CNRS, Institut des Sciences de l'Evolution, CC65, Place Eugène Bataillon, 34095 Montpellier cedex 5, France \\ Submitted April 3, 2013; Accepted October 18, 2013; Electronically published January 14, 2014 \\ Online enhancements: appendixes.
}

\begin{abstract}
Abundant empirical evidence for dispersal syndromes contrasts with the rarity of theoretical predictions about the evolution of life-history divergence between dispersing and philopatric individuals. We use an evolutionary model to predict optimal differences in age-specific reproductive effort between dispersing and philopatric individuals inhabiting the same metapopulation. In our model, only young individuals disperse, and their lifelong reproductive decisions are potentially affected by this initial event. Juvenile survival declines as density of adults and other juveniles increases. We assume a tradeoff between reproduction and survival, so that different patterns of age-specific reproductive effort lead to different patterns of aging. We find that young immigrant mothers should allocate more resources to reproduction than young philopatric mothers, but these life-history differences vanish as immigrant and philopatric individuals get older. However, whether the higher early reproductive effort of immigrants results in higher fecundity depends on the postimmigration cost on fecundity. Dispersing individuals have consequently a shorter life span. Ultimately, these life-history differences are due to the fact that young dispersing individuals most often live in recently founded populations, where competition is relaxed and juvenile survival higher, favoring larger investment in offspring production at the expense of survival.
\end{abstract}

Keywords: dispersal syndromes, aging, reproductive effort, colonizer.

\section{Introduction}

Dispersal is a major trait for the evolution and dynamics of spatially structured populations (Benton and Bowler 2012). A better understanding of dispersal processes is particularly critical for the forecasting of the consequences of habitat fragmentation, global warming, and exotic species introduction (Baguette et al. 2012; Le Galliard et al. 2012; Travis and Dytham 2012). Empirical studies show that dispersers are not a random subset of their source population, differing from philopatric individuals in morphology, behavior, physiology, and life history (Clobert et

\footnotetext{
* Corresponding author: olivier.cotto@univ-montp2.fr.
}

Am. Nat. 2014. Vol. 183, pp. 384-393. (C) 2014 by The University of Chicago. 0003-0147/2014/18303-54583\$15.00. All rights reserved.

DOI: $10.1086 / 675064$ al. 2004). Phenotypic differences between dispersers and philopatric individuals can strongly alter the consequences of dispersal for population dynamics, range expansion, community dynamics, and effective gene flow and its fitness consequences. However, predicting the phenotypes associated with dispersal is not an easy task, given the high variability in dispersal syndromes (see reviews about dispersal syndromes in Clobert et al. 2009; Bonte et al. 2012; Ronce and Clobert 2012).

In this article, our aim is to better understand the evolutionary forces that shape life-history differences between dispersers and philopatric individuals. Dispersers often exhibit different life-history traits than philopatric individuals, but the sign and magnitude of correlations between dispersal and life-history traits vary widely both between and within species (Belichon et al. 1996; Ronce and Clobert 2012). For example, dispersers have a higher survival rate than residents in the great tit (Parus major; Clobert et al. 1988) but a lower survival rate in the damselfly Calopteryx splendens (Chaput-Bardy et al. 2010). Part of such inconsistency may be explained by the many methodological issues affecting the estimation of fitness components of philopatric and dispersing individuals in nature (Belichon et al. 1996; Doligez and Part 2008).Variation in dispersal syndromes might also be due to the fact that differences in life-history traits between dispersing and philopatric individuals vary with age. For instance, in water striders winged females have a shorter pre-oviposition period than wingless females before diapause, but the trend reverses after diapause (Fairbairn 1988). In the Glanville fritillary butterfly (Melitaea cinxia), lifetime reproductive success is similar for females originating from recently founded and longer-established populations, despite their marked difference in mobility (Saastamoinen 2007). However, clutch size early in life is higher for females from recent populations, while the reverse trend is observed for older females (Hanski et al. 2006; Bonte and Saastamoinen 2012).

The interpretation of dispersal syndromes is also com- 
plicated by their multiple proximal and ultimate causes (Clobert et al. 2009; Ronce and Clobert 2012). In particular, life-history differences between philopatric and dispersing individuals could be explained because (i) energetic investment in dispersal trades off with energetic allocation to other traits, and more generally, dispersal is a costly, time-consuming, risky behavior (Bonte et al. 2012); and (ii) selection on dispersers' life-history traits differs from that on philopatric individuals. During each stage of the dispersal process, that is, departure, transience, and settlement, dispersers are indeed likely to experience new environments and thus different selective pressures than residents. Several theoretical studies have explored how divergent selection could shape phenotypes of philopatric and dispersing individuals. For example, Kisdi et al. (2012) predicted that different body size would be optimal for dispersing and philopatric individuals when transience is costly (see also Bonte and de la Peña 2009). Dispersing individuals are on average less related to other individuals in their breeding patch than philopatric individuals are, which selects for higher aggressiveness and less cooperation in dispersers (Perrin and Lehmann 2001; El Mouden and Gardner 2008; Wild and Fernandes 2009) as well as for less female-biased (Taylor and Crespi 1994) and less dispersive offspring in the progeny of dispersing parents (Asaduzzaman and Wild 2012). These predictions have been supported by data (e.g., Duckworth 2008 for aggressiveness; Taylor and Crespi 1994 for sex ratio; MacKay and Wellington 1977 for offspring dispersal). Empirical evidence for association between dispersal and survival and fecundity however contrasts with the rarity of theoretical predictions about the evolution of life-history divergence between dispersing and philopatric individuals (Ronce and Clobert 2012). Lemel et al. (1997) investigated the consequences of different life histories for residents and dispersers on the evolution of dispersal in a two-patch system and found that higher dispersal rates evolved when dispersing individuals have a higher survival compensating for the fecundity cost due to transience. However, which life-history strategy was optimal in dispersing and philopatric individuals was not explored.

Avoidance of high densities is one of the major drivers of dispersal, both from a proximate and evolutionary point-of-view (Lambin et al. 2001; Clobert et al. 2004; Ronce 2007). Dispersing and philopatric individuals are then likely to experience different levels of intraspecific competition. In particular, when dispersal leads to the colonization of new sites, as in a metapopulation with frequent patch turnover, dispersing individuals may encounter very different demographic conditions in newly colonized sites, favoring the emergence of a "colonizer syndrome" associating high mobility to rapid development, early reproduction, and high fecundity (Baker and
Stebbins 1965; Ronce and Olivieri 2004). To our knowledge, no study has investigated whether different life-history strategies would be favored by selection in dispersing and philopatric individuals depending on the level of intraspecific competition experienced after dispersal. Most theoretical studies about colonizer syndromes due to intraspecific competition instead examined covariation between mean dispersal rate and mean survival or fecundity at the scale of the whole metapopulation, reaching widely different conclusions (Ronce and Olivieri 2004; Clobert et al. 2009; Ronce and Clobert 2012). For instance, Ronce and Olivieri (1997) predict a positive correlation between life span and dispersal, while Crowley and McLetchie (2002) predict a negative correlation between these two traits.

Our aim here is to derive theoretical predictions about life-history differences between philopatric and dispersing individuals in the same metapopulation, as empirical data on dispersal syndromes is generally documented at this scale. We are particularly interested in the variation of dispersal syndromes with age and focus on the case of metapopulations with relatively frequent extinction and recolonization, as is the case in Glanville fritillary butterfly. We build on recent theoretical work on the evolution of age-specific reproductive effort in a metapopulation (Cotto et al. 2013), modifying our model to allow dispersing and philopatric individuals to express different reproductive tactics. In our model, only young individuals disperse and their lifelong reproductive decisions are potentially affected by this initial event. We assume a tradeoff between reproduction and survival, so that different patterns of age-specific reproductive effort lead to different patterns of aging. Juvenile survival declines as density of adults and other juveniles increases. We investigate whether dispersers and residents should have a different optimal strategy of age-specific reproductive effort, resulting in different age-specific fecundity and survival.

\section{Methods}

The model is an extension of that of Cotto et al. (2013). For simplicity, we assume that reproduction is asexual. We used adaptive dynamics methods to find the optimal strategy of resource allocation to reproduction at each age for philopatric and dispersing individuals, as in Cotto et al. (2013), but we allow reproductive effort to vary not only with age but also with migratory status. Detailed derivations are given in appendixes A and B (apps. A-C available online) and we just recall here the main assumptions (see Ronce and Olivieri 1997; Ronce et al. 2000, 2005 for details). The model is spatially implicit. We consider a metapopulation composed of an infinite number of patches. Each population is characterized by its age $i$, the time spent 
since its foundation. At each time step, a population has a probability $e$ to become extinct. When an extinction event occurs, the species of interest disappears from the patch. The patch is empty and is available for colonization. Only juveniles colonize empty patches where they can settle and reproduce if no extinction event occurs. We assume that the lifetime of a population is limited, for example, because of successional replacement or any other type of ephemeral habitat. When a population reaches its maximal age $i_{\max }$, it becomes extinct and the patch remains uncolonizable until it is disturbed again with a probability $e_{i \max +1}$. The metapopulation at equilibrium is characterized by a stable age structure (Olivieri et al. 1995).

The life cycle is as follows: (1) reproduction, (2) juvenile dispersal, (3) death of established adults, and (4) juvenile recruitment. Only juveniles disperse according to an island model of migration, each offspring having a probability $d$ to disperse, with a fraction $c$ dying during migration. Surviving dispersers immigrate in empty and occupied patches with a probability equal to the respective frequency of the different types of patches in the metapopulation. In the current version of the model, this dispersal rate is fixed. First reproduction occurs after one time step and, for convenience of numerical computations, life span is limited to $x_{\max }$. We checked that none of our qualitative conclusions were affected when varying the maximum life span of individuals or populations. The demography of populations is regulated through juvenile survival, which declines as the local density of other juveniles and adults in the patch increases (see app. A). Adult survival and fecundity are not affected by local density.

We assume that the decision to disperse when juvenile affects all subsequent reproductive decisions in an individual's lifetime. Among reproductive adults, we therefore distinguish immigrant individuals, which have dispersed when juvenile and established in a different patch from their natal patch, and philopatric individuals, which stayed in their natal patch. The offspring, dispersing or philopatric, and their mother have then the same genotype (asexual reproduction). Consequently, any phenotypic difference between dispersing and philopatric offspring born to the same mother results from phenotypic plasticity only, juveniles developing into either disperser or philopatric phenotypes. We look for the optimal phenotypic combinations that maximize the transmission of genotypes producing such two types of offspring.

Let index $m$ denote the migratory status of an individual, with $m=p$ for philopatric individuals and $m=d$ for dispersing individuals. Life history is modeled through the allocation of resources to reproduction versus maintenance. We note $R_{m, x}$ the proportion of resources allocated to reproduction (reproductive effort) at age $x$ by an in- dividual with the migratory status $m$. Reciprocally, the proportion allocated to maintenance is $1-R_{m, x}$. The allocation of resource determines the fecundity at age $x$, $f_{m, x}$, and the probability of survival from age $x$ to age $x+$ $1, s_{m, x}$ :

$$
\begin{aligned}
& s_{m, x}=S_{m, x}\left(1-R_{m, x}\right)^{\beta}, \\
& f_{m, x}=F_{m, x} R_{m, x}^{\beta},
\end{aligned}
$$

where $S_{m, x}$ is the maximal survival probability at age $x$ and $F_{m, x}$ is the maximal fertility at age $x$ for individuals with the migratory status $m$. The effect of resource allocation at age $x$ does not affect life-history traits at later ages ("income breeder" life cycle; Drent and Daan 1980). Alternative assumptions about the trade-offs between survival and fecundity affected the optimal pattern of agespecific reproductive effort but without affecting qualitatively our results. The coefficient $\beta$ determines the shape of the trade-off curve between survival and fecundity at age $x$. In numerical explorations, this coefficient is smaller than one, so that the trade-off between reproduction and survival is concave allowing the selection of intermediate reproductive effort (Schaffer 1974; Cotto et al. 2013). We assume that the maximal fecundity and maximal survival rate of philopatric individuals, which we note with $F_{\mathrm{p}}$ and $S_{\mathrm{p}}$, respectively, are constant with age. We further assume that immigrant individuals can have reduced maximal fecundity and/or survival with respect to philopatric individuals, and allow such a cost of dispersal to vanish with increasing age. Transience could reduce the capacity of recent immigrants to survive or reproduce after their settlement. The maximal survival rate of an immigrant with age $x$ is

$$
S_{d, x}=S_{\mathrm{p}}\left(1-\frac{1}{\gamma_{\mathrm{s}} \exp [\mathrm{x}]}\right),
$$

where $\gamma_{\mathrm{s}}$ is a parameter regulating the duration and intensity of this dispersal cost (see fig. 2). Similarly, the maximal fecundity of an immigrant at age $x$ is

$$
F_{d, x}=F_{\mathrm{p}}\left(1-\frac{1}{\gamma_{\mathrm{f}} \exp [\mathrm{x}]}\right) \text {. }
$$

\section{Analytical Results}

When the reproductive effort strategy at age $x$ is optimal, any gain in reproductive value due to the production of extra offspring will be exactly offset by the loss of expected future reproductive value as a surviving adult. A strategy of intermediate reproductive effort expressed by individual with migratory status $m$ and age $x$ corresponds to such a singular evolutionary point if (see app. B): 


$$
\begin{gathered}
{\left[(1-d) \tilde{\nu}_{\text {philo }}(x, m)+d \tilde{v}_{\text {disp }}\right] \frac{d f_{m, x}}{d R_{m, x}}} \\
+\tilde{v}(x+1, m) \frac{d s_{m, x}}{d R_{m, x}}=0 .
\end{gathered}
$$

where $\tilde{v}(x+1, m)$ is the average reproductive value of an adult with age $x+1$ and with migratory status $m$, $\tilde{v}_{\text {philo }}(x, m)$ is the average reproductive value of a philopatric juvenile born to a mother with age $x$ and with migratory status $m$, and $\tilde{v}_{\text {disp }}$ is the average reproductive value of dispersing offspring. The first two quantities differ between philopatric and immigrant mothers. In particular, the reproductive value of a philopatric offspring born to an individual with migratory status $m$ and age $x$ depends on the age the population where such an adult reproduces. Reproductive value of a philopatric offspring should in particular be higher if its parent reproduces in a patch with a low number of competitors, where juvenile survival is high. The average reproductive value of a philopatric offspring thus depends on the distribution of adults across populations with different ages (see app. B). This distribution varies depending on both the age of the adult and its migratory status (apps. A, B). The reproductive values in equation (3) were computed numerically, following the same procedure as in Cotto et al. (2013), until we found a set of reproductive efforts satisfying equation (3) for each age and migratory type. The numeric procedure we used ensures that the strategies found are also convergence stable (see Cotto et al. 2013), and we verified that these strategies corresponded to fitness maxima.

\section{Numerical Results}

Figure 1 shows that there are more dispersing adults in young populations than in old populations. In particular, adults that did not disperse when juvenile necessarily reproduce in populations that have been founded for at least two time steps, while only young dispersers reproduce in populations founded for only one time step. The number of philopatric individuals increases with the time since colonization (fig. 1A). The age structure of individuals also changes with the age of the population. Young individuals are more numerous in recently founded populations, whereas old individuals prevail in older populations. This is due to our assumption of juvenile dispersal but also to the fact that juvenile survival rate is higher in recently founded populations where density is lower and the population is growing. Indeed, when the number of founders is small, the juvenile survival rate is high in young populations and rapidly decreases in older populations (fig. $1 B)$. The changes in juvenile survival with the age of the population are negatively correlated with the changes in
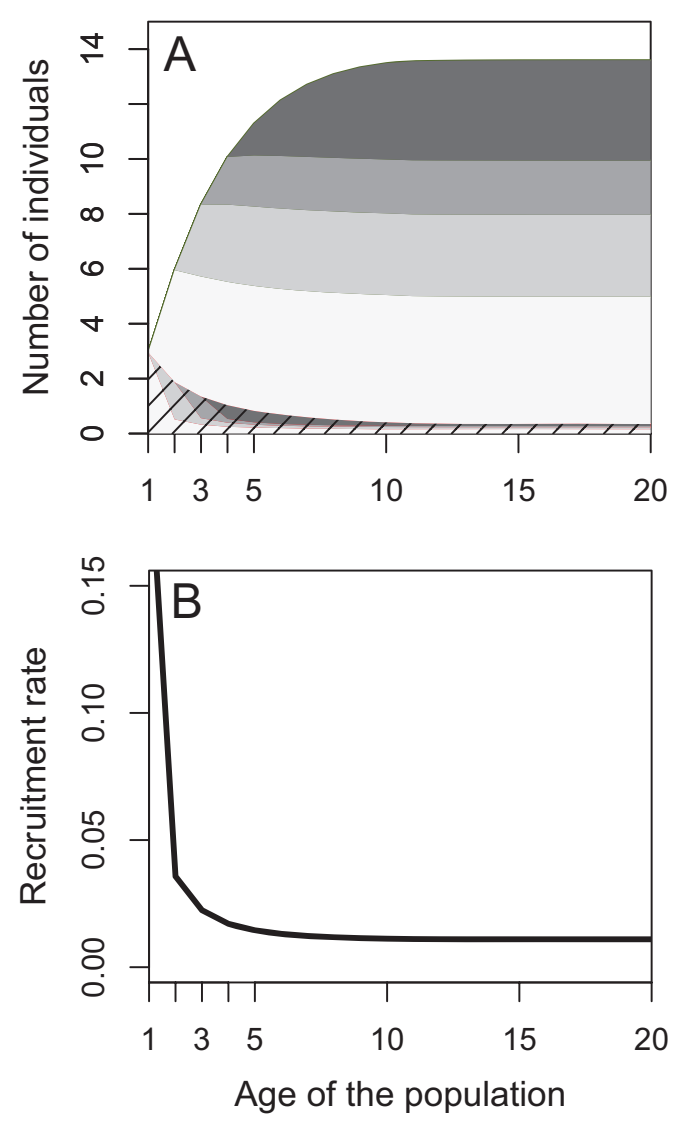

Figure 1: Number of individuals $(A)$ and recruitment rate $(B)$ function of the age of the population. Number of dispersing individuals (striped) and philopatric individuals (unstriped); tones represent the age classes. The brighter tone is the age class 1 , followed by age classes 2, 3, and older than 3 (darkest tone). The full line represents change in the juvenile survival rate with the age of the population. Other parameters: $d=0.1, e=0.1, S_{m}=0.9, F_{m}=20, k_{0}=$ $k_{x}=0.1, c=0.5, \sigma_{0}=0.9, i_{\max }=20, x_{\max }=10$, no postimmigration cost, $\gamma_{\mathrm{s}}=\gamma_{\mathrm{f}}=100$.

density. This effect can be exaggerated when older individuals monopolize more resources reducing juvenile survival (not shown).

Only young immigrant adults benefit from these favorable ecological conditions following colonization because they are frequent in such populations. Philopatric individuals start to reproduce in populations where juvenile survival rate is already much lower due to growing density and live on average in populations with a lot of competition. In consequence, according to equation (3), we would expect young immigrants to invest more energy in production of juveniles than young philopatric adults do.

In accordance with our expectation, immigrant and philopatric individuals have different optimal age-specific 
reproductive effort at the beginning of their life. Dispersing individuals should spend most of their resources in reproduction when they are young contrarily to philopatric individuals (fig. 2A, 2B). The optimal reproductive effort increases at the end of life for both migratory types, consistently with previous results (Schaffer 1974). This difference in reproductive effort in early ages results in a lower average life span for immigrants (fig. $2 E, 2 F$ ). However, the difference in age-specific fecundity between immigrant and philopatric individuals depends on our assumptions about the cost of transience on life-history traits. Young immigrant mothers have a higher fecundity than young philopatric mothers when dispersal has no or a small effect on the age-specific maximal fecundity (fig. $2 C)$. When dispersal reduces the reproductive performance of individuals after settlement, young philopatric mothers can have a higher fecundity than young immigrant mothers, despite a lower reproductive effort (fig. 2B, 2D). Philopatric mothers then have higher lifetime reproductive success than immigrant mothers (higher fecundity and longer expected life span; fig. $2 F$ ) whereas, in the absence of transience cost on fecundity, the reverse trend can be found (not shown). Depressed maximal survival of immigrants does not change qualitatively these predictions (not shown).

Figure 3 shows the proportional differences in age-spe-
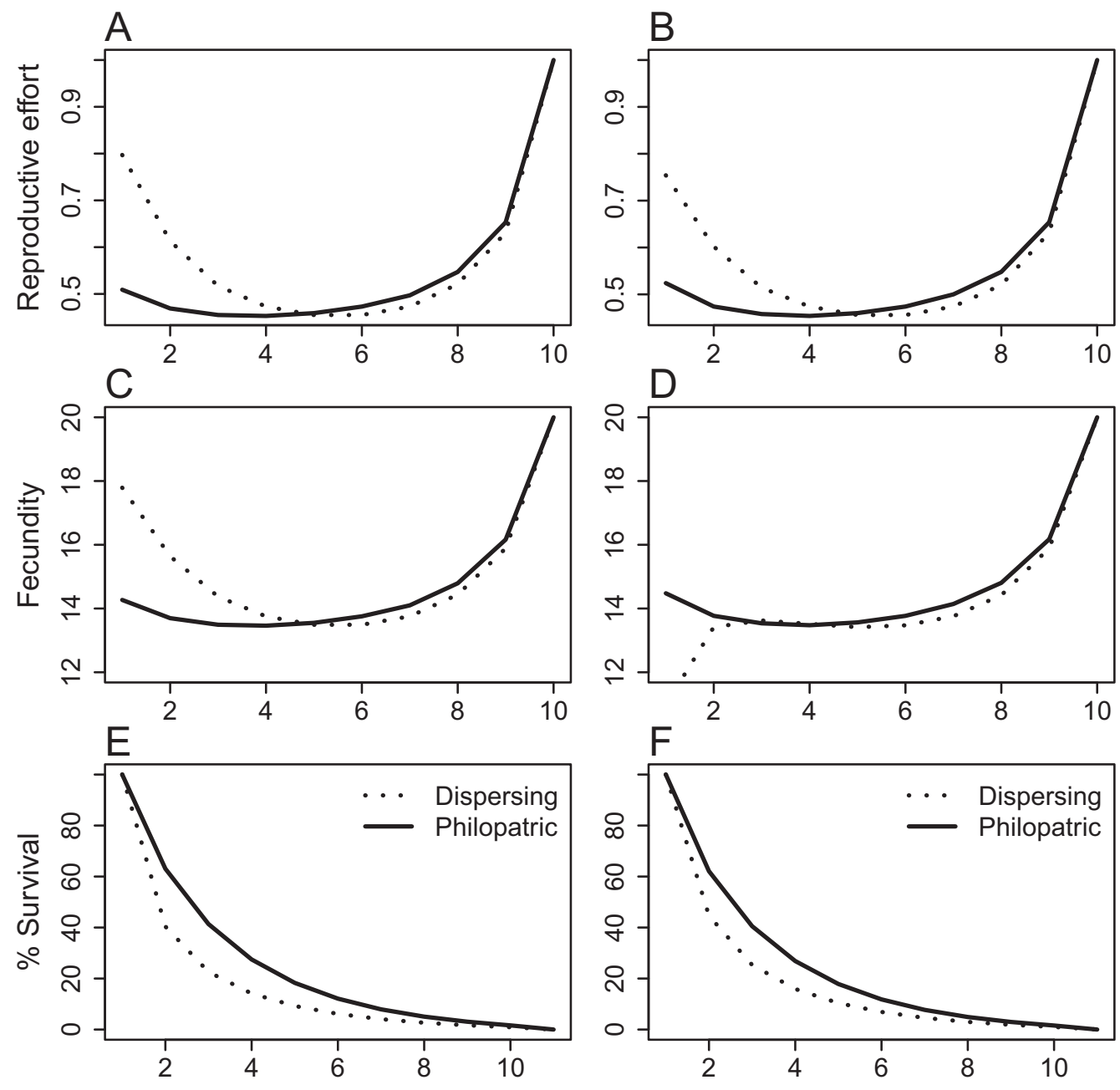

Age of the individual, $x$

Figure 2: Age-specific reproductive effort $(A, B)$, fecundity $(C, D)$, and fraction surviving $(E, F)$ of dispersing and philopatric individuals. $A, C, E$, No postimmigration cost, $\gamma_{\mathrm{s}}=\gamma_{\mathrm{f}}=100 . B, D, F$, Transience reduces individual reproductive performances, $\gamma_{\mathrm{f}}=1$, but not survival after immigration, $\gamma_{\mathrm{s}}=100$. Other parameters: $d=0.1, e=0.1, S_{m}=0.9, F_{m}=20, k_{0}=k_{x}=0.1, c=0.5, \sigma_{0}=0.9, i_{\max }=$ $20, x_{\max }=10$. 


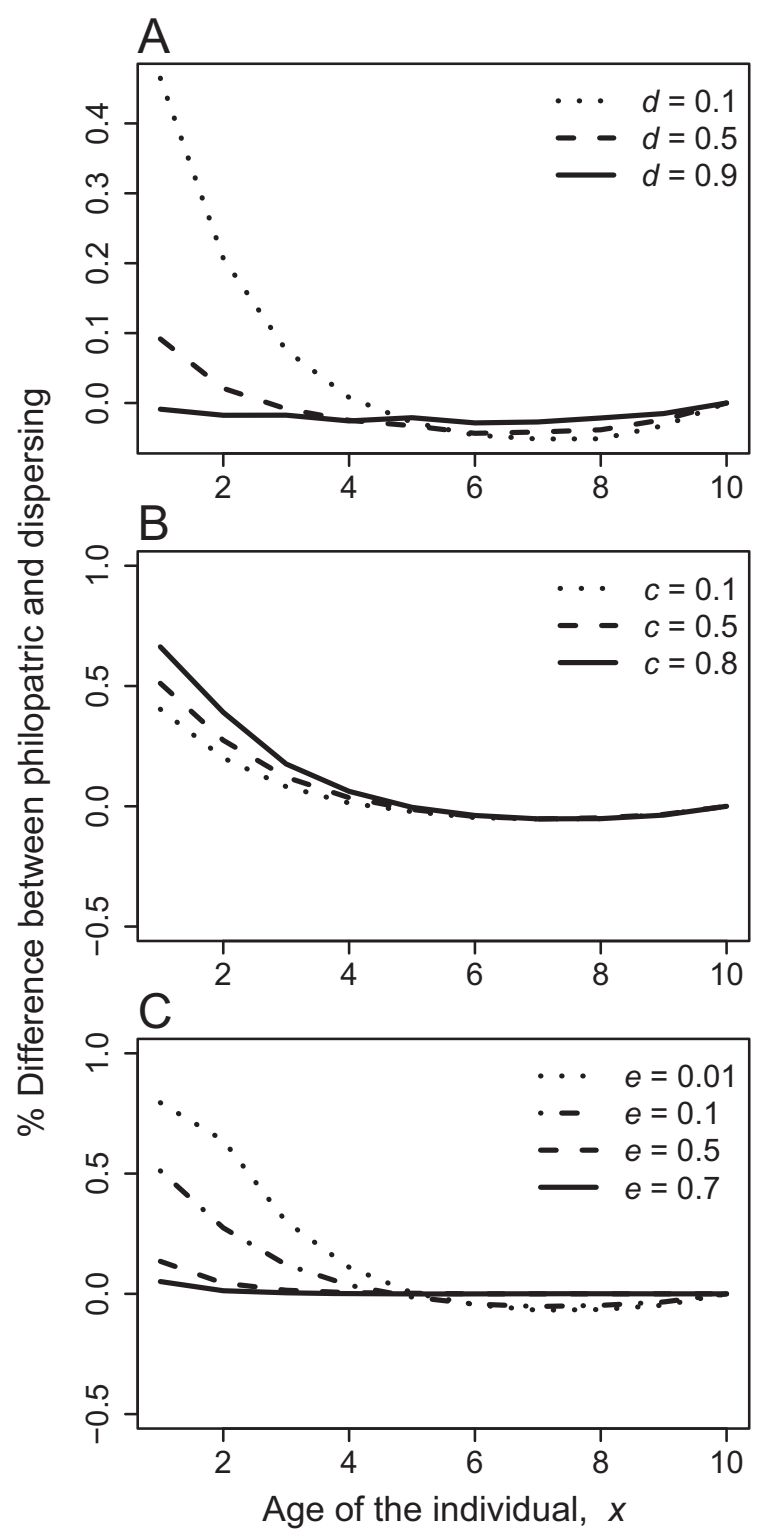

Figure 3: Proportional difference $\left(\left[R_{d, x}-R_{p, x}\right] / R_{d, x}\right)$ in age-specific reproductive effort between dispersing and philopatric adults for various dispersal rates $(A)$, costs of dispersal $(B)$, and extinction rates (C). When not specified, $d=0.1, e=0.1, c=0.5$. Other parameters: $S_{m}=0.9, F_{m}=20, k_{0}=k_{x}=0.1, \sigma_{0}=0.9$, no postimmigration cost, $\gamma_{\mathrm{s}}=\gamma_{\mathrm{f}}=100, x_{\max }=10, i_{\max }=20$.

cific reproductive effort between immigrant and philopatric individuals of the same metapopulation for various dispersal rates, costs of dispersal, and extinction rates. When the dispersal rate increases, the age-specific reproductive effort of dispersing and philopatric individuals converge (fig. 3A). The effect of high dispersal rate is twofold. First, when most juveniles disperse, the recruitment conditions in the parental patch do not matter much. The average recruitment prospects of (mostly dispersing) juveniles are then the same whatever the status of their parent. Second, when dispersing individuals are numerous, the number of founders recolonizing disturbed patches is large. The patches are then crowded immediately after colonization so that the juvenile survival rate is low in all populations. Therefore, the philopatric offspring of dispersing and philopatric individuals have similar recruitment rates. Conversely, when the dispersal rate is low, there are fewer juveniles colonizing empty patches so that the recruitment opportunities in young populations are higher, selecting for higher reproductive effort in young dispersing mothers. Accordingly, if a lot of juveniles die during dispersal, dispersing individuals invest more in reproduction relative to philopatric individuals (fig. $3 B$ ). The effect of increasing the cost of dispersal is lower than the effect of decreasing the dispersal rate, because the former affects the number of colonizers but not the number of philopatric offspring. Increasing the extinction rate also a twofold effect. On the one hand, it increases the number of empty patches and hence decreases the number of colonizers per patch. On the other hand, high extinction rates increase extrinsic mortality for both philopatric and immigrant adults, thus selecting for high reproductive effort (Ronce and Olivieri 1997; Cotto et al. 2013) in both types of individuals. The latter effect dominates, and we find that high extinction rates reduce the difference in reproductive effort between the two migratory types (fig. 3C).

\section{Discussion}

Differences in life history between dispersing and philopatric individuals are often understood as resulting from the costs of dispersal (Bonte et al. 2012; Ronce and Clobert 2012). Dispersal can be associated with lower or delayed fecundity and lower survival, which is interpreted to result from the cost of transience or developmental constraints (Bonte et al. 2012). However, in this study we show that differences in life-history between dispersing and philopatric individuals could also be selected for because both types do not live on average in the same environments.

We modeled a metapopulation with extinction-recolonization (Levins 1969) where only juveniles disperse and populations grow after foundation. Young individuals are then overrepresented in young populations. When the number of colonizers is low, recruitment opportunities are abundant for juveniles in young populations but not in old populations. Dispersing individuals start to reproduce in a favorable environment, whereas philopatric individuals start to reproduce later in more crowded populations. Thus, depending on their age and dispersal history, individuals do not live on average in the same environment. As a result, dispersing adults should allocate more resource 
to reproduction at the beginning of their life, which allows exploiting favorable local opportunities following colonization. In this respect, the dispersal syndrome predicted by our model could be described as a colonizer syndrome (Baker and Stebbins 1965). Low dispersal, high cost of dispersal, and low initial performances reduce the population size at foundation and consequently favor higher reproductive effort in young dispersing adults. However, as they age, immigrant and philopatric individuals live in similar environments. The differences in life-history decisions between philopatric and dispersing thus vanish as individuals get older.

An interesting case is when dispersal entails a cost on initial individual performances due for example to transience (Belichon et al. 1996; Bonte et al. 2012). In this case, we found that philopatric individuals can have a higher average survival and fecundity than dispersing individuals despite the latter having a higher reproductive effort. This emphasizes the importance to distinguish fecundity from reproductive effort. For instance, even though the oogenesis-flight syndrome is widespread in insects (e.g., Roff and Fairbairn 2001), dispersing individuals sometimes reallocate resource from flight muscles via histolysis after settlement (Harrison 1980; Derr et al. 1981 in Bonte and Saastamoinen 2012; see also a review in Rankin and Burchsted 1992 in favor of this idea). Measuring reproductive effort remains a challenge (Clutton-Brock 1984; Descamps et al. 2007; Fletcher et al. 2013). Measuring daily energy expenditure, oxidative damage, and antioxidant protection, along with fitness components in each age class, could allow disentangling the role of constraints and life-history decisions in shaping dispersal syndromes.

Our model ignores kin competition, an important driver of dispersal in small populations (Frank 1986; Poethke et al. 2007), predicted to affect the evolution of dispersal behavioral syndromes (El Mouden and Gardner 2008), as well as the evolution of reproductive effort (Pen 2000). In a structured population model, Pen (2000) found in particular that the optimal reproductive effort increases with kin competition. As dispersers may be less related to their neighbors than philopatric individuals, one would then predict that the former should have a lower reproductive effort. However, predictions are less straightforward in a metapopulation where extinction-recolonization dynamics may alter patterns of kin competition for dispersing and philopatric individuals in a complex manner. Computing kin selection measures in structured metapopulations with a large number of demographic classes is technically difficult (Rousset and Ronce 2004), preventing a simple analytical approach of the question. To check on the robustness of our predictions in the presence of kin competition, we performed individual-based simulations of a model with otherwise similar assumptions as in our analytical model (app. C). Our preliminary exploration shows that our qualitative prediction of a higher reproductive effort in young dispersers in a metapopulation still holds, even when patch carrying capacity is low and kin competition is potentially strong (app. C).

Few empirical studies investigating dispersal syndromes describe trait variation with age (see, however, Auld and Charmantier 2011), as most studies report the total number of offspring (Hansson et al. 2004), the average life span (Hansson et al. 2004), or the average daily fecundity (Chaput-Bardy et al. 2010) of dispersing and philopatric individuals. Our model suggests that dispersal syndromes should vary with the age of individuals, which has been investigated in few empirical studies. Ignoring the variation of life histories with age could thus potentially obscure the detection of dispersal syndromes.

We here discuss those few empirical results where agespecific variation in life-history traits in immigrant and philopatric individuals has been described. We hope that this study will stimulate the reanalysis of more data sets of this kind. Our predictions should be more relevant for species living in metapopulations where at least some dispersers settle in patches with low competition. The Glanville fritillary living in the Aland islands system is a good example of such species. Each year many sites are colonized and become extinct (Bonte and Saastamoinen 2012). In new populations, there are mostly individuals with dispersing genotypes (Haag et al. 2005). Females from new populations initiate reproduction at younger age, have higher mating frequency and most importantly lay more eggs when they are young than females from old populations (Hanski et al. 2006). Conversely, old females from old populations lay more eggs than old females from new populations. This pattern of variation in fecundity is consistent with the interaction between individual age and dispersal propensity predicted by our model when the cost of dispersal on fecundity is not too high. Finally, Glanville fritillary females from new populations have a lower life span than females from old populations (Hanski et al. 2006), which is also consistent with our predictions if we assume that most of these females are dispersers. Preliminary results indicate that common lizards (Zootoca vivipara) having dispersed out of their mother territory as juveniles show higher early reproductive effort than philopatric individuals and that these differences vanish as they age (O. Cotto et al., unpublished manuscript), consistent with our predictions. There is further evidence that individuals dispersing because of kin competition in that species are larger and better colonizers than philopatric individuals or those individuals dispersing for other reasons (Le Galliard and Clobert 2003; Cote and Clobert 2007). 
Dispersal phenotypes and syndromes often depend on the context and motivation for dispersal (Clobert et al. 2004) and more generally vary with habitat selection strategies (Le Galliard and Clobert 2003; Cote and Clobert 2007), with probably different optimal life histories after settlement. Our study suggests that we expect difference in life histories between colonizers and philopatric individuals but not necessarily between the latter and other types of dispersers. For instance, in great tits, immigrant females have a lower fecundity than resident females when they are young but they live on average longer (Clobert et al. 1988; Tully et al. 2004; Auld and Charmantier 2011), which could be interpreted as young immigrant tits having a lower reproductive effort than residents, contrary to our prediction. In this last case study, the immigrant individuals had established in an already occupied and dense population: whether they are representative of other dispersing individuals (e.g., those colonizing empty habitats) is an open question.

An interesting consequence of our findings is that the sign of the predicted covariation of life-history traits with dispersal changes depending on the scale of the study. When comparing different metapopulations with different dispersal rates but otherwise similar assumptions as in this study, reproductive effort correlates negatively with dispersal (Ronce et al. 2000). This happens because higher dispersal results in higher density and lower juvenile survival after colonization, selecting for decreasing investment into reproduction. In this study, we conversely found a positive correlation between dispersal and reproductive effort when contrasting life-history strategies of dispersing and philopatric individuals in the same metapopulation. This shift in the sign of the correlation between dispersal and reproductive effort across scales can be a source of inconsistency among empirical studies and between empirical studies and theoretical predictions interested in dispersal syndromes (Ronce and Clobert 2012).

Our study showed that, in a metapopulation with extinction-recolonization, dispersing and philopatric individuals should have a different schedule of resource allocation. This schedule of resource allocation is driven by the favorable ecological conditions encountered in recently colonized populations and by the fact that, depending on their dispersing status and age, not all individuals encounter these conditions. Our model highlights the need to study dispersal syndromes at different scales both empirically and theoretically, considering explicitly how such syndromes vary with age.

\section{Acknowledgments}

We thank J. Clobert and I. Olivieri for helpful discussions and comments and T. Day, A. Gardner, J. F. Wilkins, and an anonymous reviewer for help in improving our paper and pointing out important literature. O.C. acknowledges support from the Ministry of Research through a $\mathrm{PhD}$ scholarship. A.K. acknowledges funding from the European Research Area Network BiodivERsA TipTree project (ANR-12-EBID-0003). This is publication ISEM 2013-152 from the Institut des Sciences de l'Evolution, Montpellier. This work was supported by the French Agropolis Fondation (Réseau thématique de recherche avancée Montpellier, BIOFIS project 1001-001) and Agence Nationale de la Recherche, Génétique et Evolution de la Spécialisation (2010 BLAN 1715).

\section{Literature Cited}

Asaduzzaman, S. M., and G. Wild. 2012. The evolution of dispersal conditioned on migration status. Ecology and Evolution 2:822843. doi:10.1002/ece3.99.

Auld, J. R., and A. Charmantier. 2011. Life history of breeding partners alters age-related changes of reproductive traits in a natural population of blue tits. Oikos 120:1129-1138.

Baguette, M., D. Legrand, H. Fréville, H. Van Dyck, and S. Ducatez. 2012. Evolutionary ecology of dispersal in fragmented landscape. Page 381-391 in J. Clobert, M. Baguette, T. G. Benton, and J. M. Bullock, eds. Dispersal ecology and evolution. Oxford University Press, Oxford.

Baker, H. G., and G. L. Stebbins. 1965. The genetics of colonizing species. Academic Press, New York.

Belichon, S., J. Clobert, and M. Massot. 1996. Are there differences in fitness components between philopatric and dispersing individuals? Acta Oecologica 17:503-517.

Benton, T. G., and D. E. Bowler. 2012. Linking dispersal to spatial dynamics. Page 251-265 in J. Clobert, M. Baguette, T. G. Benton, and J. M. Bullock, eds. Dispersal ecology and evolution. Oxford University Press, Oxford.

Bonte, D., and E. De La Pena. 2009. Evolution of body conditiondependent dispersal in metapopulations. Journal of Evolutionary Biology 22:1242-1251.

Bonte, D., and M. Saastamoinen. 2012. Dispersal syndromes in butterflies and spiders. Page 161-170 in J. Clobert, M. Baguette, T. G. Benton, and J. M. Bullock, eds. Dispersal ecology and evolution. Oxford University Press, Oxford.

Bonte, D., H. Van Dyck, J. M. Bullock, A. Coulon, M. Delgado, M. Gibbs, V. Lehouck, et al. 2012. Costs of dispersal. Biological Reviews 87:290-312.

Chaput-Bardy, A., A. Gregoire, M. Baguette, A. Pagano, and J. Secondi. 2010. Condition and phenotype-dependent dispersal in a damselfly, Calopteryx splendens. PLoS ONE 5:e10694. doi:10.1371/ journal.pone.0010694.

Clobert, J., R. Ims, and F. Rousset. 2004. Causes, mechanisms and consequences of dispersal. Page 307-335 in I. Hanski and O. E. Gaggiotti, eds. Ecology, genetics and evolution of metapopulations. Elsevier Academic, New York.

Clobert, J., J. F. Le Galliard, J. Cote, S. Meylan, and M. Massot. 2009. Informed dispersal, heterogeneity in animal dispersal syndromes and the dynamics of spatially structured populations. Ecology Letters 12:197-209. 
Clobert, J., C. M. Perrins, R. H. McCleery, and A. G. Gosler. 1988. Survival rate in the great tit Parus major in relation to sex, age, and immigration status. Journal of Animal Ecology 57:287-306.

Clutton-Brock, T. H. 1984. Reproductive effort and terminal investment in iteroparous animals. American Naturalist 123:212229.

Cote, J., and J. Clobert. 2007. Social personalities influence natal dispersal in a lizard. Proceedings of the Royal Society B: Biological Sciences 274:383-390.

Cotto, O., I. Olivieri, and O. Ronce. 2013. Optimal life-history schedule in a metapopulation with juvenile dispersal. Journal of Evolutionary Biology 26:944-954. doi:10.1111/jeb.12100.

Crowley, P. H., and D. N. McLetchie. 2002. Trade-offs and spatial life-history strategies in classical metapopulations. American Naturalist 159:190-208.

Derr, J. A., B. Alden, and H. Dingle. 1981. Insect life histories in relation to migration, body size, and host plant array: a comparative study of Dysdercus. Journal of Animal Ecology 50:181-193.

Descamps, S., S. Boutin, D. Berteaux, and J. M. Galliard. 2007. Female red squirrels fit Williams' hypothesis of increasing reproductive effort with increasing age. Journal of Animal Ecology 76:11921201.

Doligez, B., and T. Part. 2008. Estimating fitness consequences of dispersal: a road to "know-where"? non-random dispersal and the underestimation of dispersers' fitness. Journal of Animal Ecology 77:1199-1211.

Drent, R. H., and S. Daan. 1980. The prudent parent: energetic adjustments in avian breeding. Ardea 68:225-252.

Duckworth, R. A. 2008. Adaptive dispersal strategies and the dynamics of a range expansion. American Naturalist 172(suppl.):S4S17.

El Mouden, C., and A. Gardner. 2008. Nice natives and mean migrants: the evolution of dispersal-dependent social behaviour in viscous populations. Journal of Evolutionary Biology 21:14801491.

Fairbairn, D. J. 1988. Sexual selection for homogamy in the Gerridae: an extension of Ridley's comparative approach. Evolution 42:12121222.

Fletcher, Q. E., C. Selman, S. Boutin, A. G. McAdam, S. B. Woods, A. Y. Seo, C. Leeuwenburgh, J. R. Speakman, and M. M. Humphries. 2013. Oxydative damage increases with reproductive energy expenditure and is reduced by food-supplementation. Evolution 67:1527-1536. doi:10.1111/evo.12014.

Frank, S. A. 1986. Dispersal polymorphisms in subdivided populations. Journal of Theoretical Biology 122:303-309.

Haag, C. R., M. Saastamoinen, J. H. Marden, and I. Hanski. 2005. A candidate locus for variation in dispersal rate in a butterfly metapopulation. Proceedings of the Royal Society B: Biological Sciences 272:2449-2456.

Hanski, I., M. Saastamoinen, and O. Ovaskainen. 2006. Dispersalrelated life-history trade-offs in a butterfly metapopulation. Journal of Animal Ecology 75:91-100.

Hansson, B., S. Bensch, and D. Hasselquist. 2004. Lifetime fitness of short- and long-distance dispersing great reed warblers. Evolution 58:2546-2557.

Harrison, R. G. 1980. Dispersal polymorphisms in insects. Annual Review of Ecology and Systematics 11:95-118.

Kisdi, E., M. Utz, and M. Gyllenberg. 2012. Evolution of conditiondependent dispersal. Page 139-151 in J. Clobert, M. Baguette, T.
G. Benton, and J. M. Bullock, eds. Dispersal ecology and evolution. Oxford University Press, Oxford.

Lambin, X., J. Aars, and S. B. Piertney. 2001. Dispersal, intraspecific competition, kin competition and kin facilitation: a review of the empirical evidence. Pages 110-122 in J. Clobert, E. Danchin, A. A. Dhondt, and J. D. Nichols, eds. Dispersal. Oxford University Press, Oxford.

Le Galliard, J. F., R. Ferrière, and J. Clobert. 2003. Mother-offspring interactions affect natal dispersal in a lizard. Proceedings of the Royal Society B: Biological Sciences 270:1163-1169.

Le Galliard, J. F., M. Massot, and J. Clobert. 2012. Dispersal and range dynamics in changing climates: a review. Page 317-336 in J. Clobert, M. Baguette, T. G. Benton, and J. M. Bullock, eds. Dispersal ecology and evolution. Oxford University Press, Oxford.

Lemel, J.-Y., S. Belichon, J. Clobert, and M. E. Hochberg. 1997. The evolution of dispersal in a two-patch system: some consequences of differences between migrants and residents. Evolutionary Ecology 11:613-629.

Levins, R. 1969. Some demographic and genetic consequences of environmental heterogeneity for biological control. Bulletin of the Ecological Society of America 15:237-240.

MacKay, P. A., and W. G. Wellington. 1977. Maternal age as a source of variation in the ability of an aphid to produce dispersing forms. Research in Population Ecology 18:195-209.

Olivieri, I., Y. Michalakis, and P.-H. Gouyon. 1995. Metapopulation genetics and the evolution of dispersal. American Naturalist 146: 202-228.

Pen, I. 2000. Reproductive effort in viscous populations. Evolution 54:293-297.

Perrin, N., and L. Lehmann. 2001. Is sociality driven by the cost of dispersal of the benefits of philopatry? a role of kin-discrimination mechanisms. American Naturalist 158:471-483.

Poethke, H. J., B. Pfenning, and T. Hovestadt. 2007. The relative contribution of individual and kin selection to the evolution of density-dependent dispersal rates. Evolutionary Ecology Research 9:41-50.

Rankin, M., and J. Burchsted. 1992. The cost of migration in insects. Annual Review of Entomology 37:533-559.

Roff, D. A., and D. J. Fairbairn. 2001. The genetic basis of dispersal and migration, and its consequences for the evolution of correlated traits. Page 191-202 in J. Clobert, E. Danchin, A. A. Dhondt, and J. D. Nichols, eds. Dispersal. Oxford University Press, Oxford.

Ronce O. 2007. How does it feel to be like a rolling stone? Ten questions about dispersal evolution. Annual Review of Ecology, Evolution and Systematics 38:231-253

Ronce, O., S. Brachet, I. Olivieri, P. H. Gouyon, and J. Clobert. 2005. Plastic changes in seed dispersal along ecological succession: theoretical predictions from an evolutionary model. Journal of Ecology 93:431-440.

Ronce, O., and J. Clobert. 2012. Dispersal syndromes. Page 119-138 in J. Clobert, M. Baguette, T. G. Benton, and J. M. Bullock, eds. Dispersal ecology and evolution. Oxford University Press, Oxford. Ronce, O., and I. Olivieri. 1997. Evolution of reproductive effort in a metapopulation with local extinctions and ecological succession. American Naturalist 150:220-249.

- 2004. Life-history evolution in metapopulation. Page 227257 in I. Hanski and O. E. Gaggiotti, eds. Ecology, genetics, and evolution of metapopulations. Elsevier Academic, New York.

Ronce, O., F. Perret, and I. Olivieri. 2000. Landscape dynamics and evolution of colonizer syndromes: interactions between reproduc- 
tive effortand dispersal in a metapopulation. Evolutionary Ecology 14:233-260.

Rousset, F., and O. Ronce. 2004. Inclusive fitness for traits affecting metapopulation demography. Theoretical Population Biology 65: 127-141.

Saastamoinen, M. 2007. Mobility and lifetime fecundity in new versus old populations of the Glanville fritillary butterfly. Oecologia (Berlin) 153:569-578.

Schaffer, W. M. 1974. Optimal reproductive effort in fluctuating environments. American Naturalist 108:783-790.

Taylor, P. D., and B. J. Crespi. 1994. Evolutionarily stable strategy sex ratios when correlates of relatedness can be assessed. American Naturalist 143:297-316.

Travis, J. M., and C. Dytham. 2012. Dispersal and climate change: a review of theory. Page 337 in J. Clobert, M. Baguette, T. G. Benton, and J. M. Bullock, eds. Dispersal ecology and evolution. Oxford Unviversity Press, Oxford.

Tully, T., J. Clobert, R. H. McCleery, and C. M. Perrins. 2004. Parentoffspring competition and natal dispersal at several spatial scales in the great tit, Parus major. Oxford University Research Archive, Oxford.

Wild, G., and D. Fernandes. 2009. Investment in the public good through conditional phenotypes of large effects. Journal of Evolutionary Biology 22:927-941.

Associate Editor: Andy Gardner Editor: Troy Day

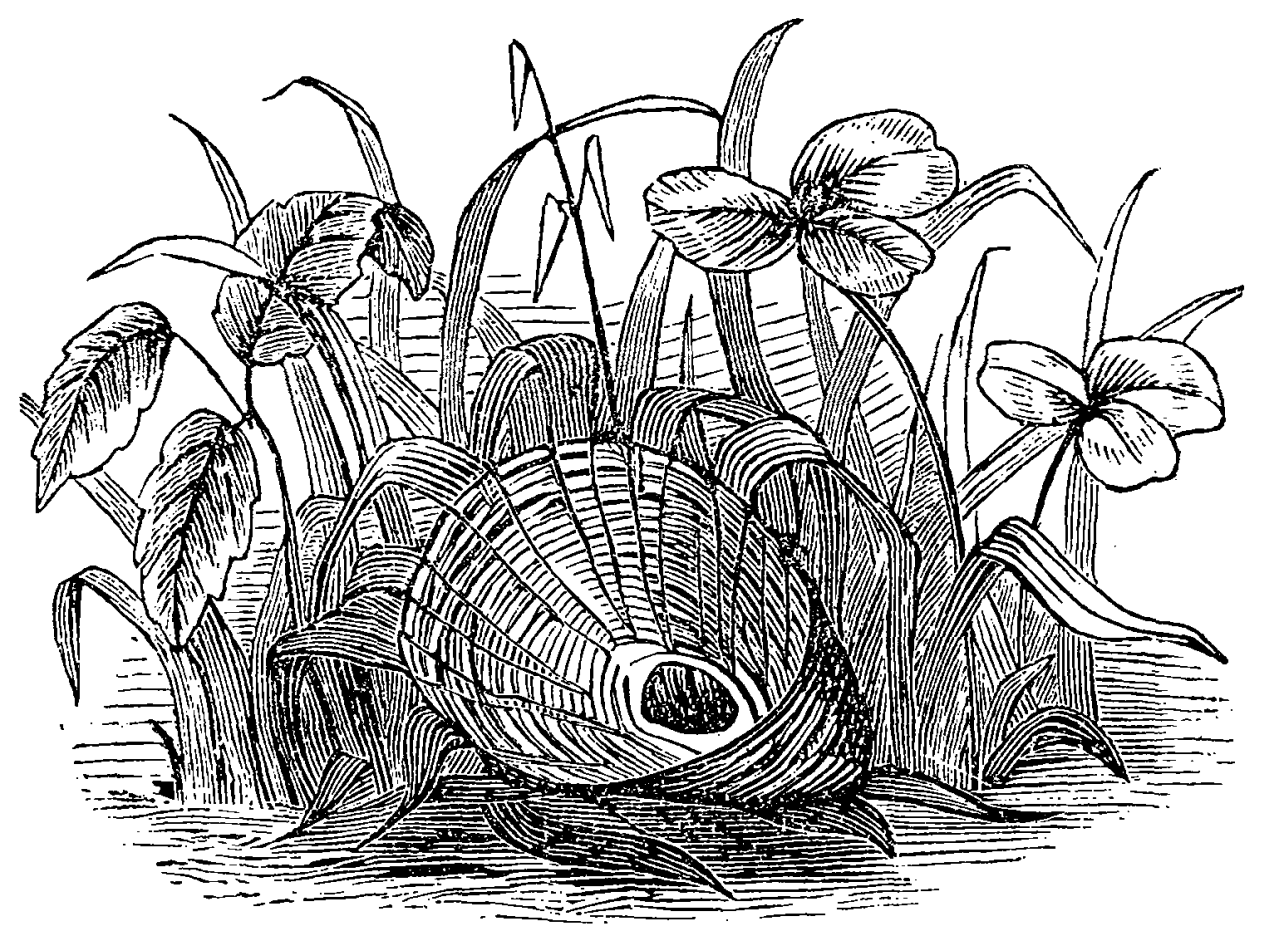

\footnotetext{
"A few days thereafter the spider made another cover, entirely unlike the winter one, more like a little room. The nest is situated in a bed of green moss, and the cover looks like a little oval mound of moss and leaves.... The base of the cover is made of acorn cups and sticks firmly held together with strands of silk, then a canopy of web is made, and over this is laid green moss, dry pine needles, bits of dry oak leaves and light sticks held fast with web. This makes a neat little upper room, the walls [of which] are smooth on the inside but rough outside. She leaves a window in the room, the object of which is apparent. She has a cocoon of eggs attached to the spinneret, and she puts herself in position to let the cocoon rest against the window where it receives the rays of the sun. For three weeks this has been her daily occupation-patiently holding the eggs in the sun." From "The Habits of a Tarantula" by Mary Treat (The American Naturalist, $1879,13: 485-489)$.
} 